\title{
THE INTERESTING SPECTRAL INTERLACING PROPERTY FOR A CERTAIN TRIDIAGONAL MATRIX*
}

\author{
CARLOS M. DA FONSECA ${ }^{\dagger}$, EMRAH KıLıÇ, AND ANTÓNIO PEREIRA ${ }^{\S}$
}

\begin{abstract}
In this paper, a new tridiagonal matrix, whose eigenvalues are the same as the Sylvester-Kac matrix of the same order, is provided. The interest of this matrix relies also in that the spectrum of a principal submatrix is also of a Sylvester-Kac matrix given rise to an interesting spectral interlacing property. It is proved alternatively that the initial matrix is similar to the Sylvester-Kac matrix.
\end{abstract}

Key words. Sylvester-Kac matrix, Tridiagonal matrices, Determinant, Eigenvalues.

AMS subject classifications. 15A15, 15A18, 65F 15.

1. Introduction. For any positive integer $n$, the $n+1$ numbers

$$
-n,-n+2,-n+4, \ldots, n-4, n-2, n
$$

are the eigenvalues of the so-called Sylvester-Kac matrix

$$
A_{n}=\left(\begin{array}{cccccc}
0 & 1 & & & & \\
n & 0 & 2 & & & \\
& n-1 & \ddots & \ddots & & \\
& & \ddots & \ddots & n-1 & \\
& & & 2 & 0 & n \\
& & & & 1 & 0
\end{array}\right) .
$$

We will call the sequence (1.1) the n-Sylvester spectrum. In the matrices throughout the text, all nonmentioned entries should be read as zero.

The tridiagonal matrix $A_{n}$ was first considered by J.J. Sylvester in 1854 in a succinct note [25] where its characteristic polynomial was conjectured. As many problems in mathematics, this was a simple problem to state but hard to prove. A definite proof to Sylvester's claim is commonly attributed to M. Kac (for both eigenvalues and eigenvectors) in his celebrated work [17], almost a century after the original statement. Notwithstanding, the Sylvester-Kac matrix has a rich history, with many proofs, in different areas, extensions, reinventions, and applications. Perhaps the most significant literature is $[1,3,6,7,9-16,18-24,26,27]$. The Sylvester-Kac matrix, is also known as Clement matrix due to the independent study of P.A. Clement in [9].

\footnotetext{
${ }^{*}$ Received by the editors on August 27, 2019. Accepted for publication on July 12, 2020. Handling Editor: Panayiotis Psarrakos. Corresponding Author: Carlos M. da Fonseca.

${ }^{\dagger}$ Kuwait College of Science and Technology, P.O. Box 27235, Safat 13133, Kuwait (c.dafonseca@kcst.edu.kw), and University of Primorska, FAMNIT, Glagoljsaška 8, 6000 Koper, Slovenia (carlos.dafonseca@famnit.upr.si).

${ }^{\ddagger}$ TOBB University of Economics and Technology, Mathematics Department, 06560 Ankara, Turkey (ekilic@etu.edu.tr).

$\S$ Departamento de Matemática, Universidade de Aveiro, 3810-193 Aveiro, Portugal (antoniop@ua.pt).
} 
As mentioned in [26], there are many generalizations of Sylvester's claim. Some have been established by Askey and Wilson [2] and remain largely open. Interestingly and somehow surprisingly, there is a close connection with Krawtchouk polynomials, which are polynomials orthogonal with respect to a binomial distribution. On the other hand, there is also an intimate relation with graph theory, namely to problems about distance regular graphs [4, p. 246].

Let us consider the tridiagonal matrix

$$
\tilde{H}_{n}=\left(\begin{array}{cccccc}
0 & 1 / 2 & & & & \\
\sigma_{n, n} & 0 & 1 / 2 & & & \\
& \sigma_{n-1, n} & \ddots & \ddots & & \\
& & \ddots & \ddots & 1 / 2 & \\
& & & \sigma_{2, n} & 0 & 1 \\
& & & & \sigma_{1, n} & 0
\end{array}\right),
$$

where, for any $k=0,1, \ldots, n-1$, we define

$$
\sigma_{k, n}=\frac{(n-k+1)(n+k)}{2} .
$$

That is, $\sigma_{k, n}$ is the sum of all nonnegative integers from $k$ to $n$. Clearly, the spectrum of $\tilde{H}_{n}$ is the same as the matrix

$$
H_{n}=\left(\begin{array}{cccccc}
0 & \frac{1}{2} & & & & \\
\frac{2 n}{2} & 0 & \frac{2}{2} & & & \\
& \frac{2 n-1}{2} & \ddots & \ddots & & \\
& & \ddots & \ddots & \frac{n-1}{2} & \\
& & & \frac{n+2}{2} & 0 & n \\
& & & & \frac{n+1}{2} & 0
\end{array}\right) .
$$

In this paper, we show by two distinct ways that $H_{n}$ shares the same spectrum as the Sylvester-Kac matrix $A_{n}$, i.e., the $n$-Sylvester spectrum. What is particularly interesting in this matrix is that when we delete the last row and column of $H_{n}$, we get a principal submatrix whose eigenvalues form the $(n-1)$ Sylvester spectrum. This means that Cauchy's interlacing theorem satisfies

$$
-n<1-n<2-n<\cdots<n-2<n-1<n .
$$

Since we know all the spectral properties of the Sylvester-Kac matrix, this matrix is very useful as what is known as test matrix. In general, test matrices are used to evaluate the accuracy of matrix inversion programs since the exact inverses are known (cf. e.g. [5,21] and references therein). Recently, Coelho, Dimitrov, and Rakai in [8] suggested a method for a fast estimation of the largest eigenvalue of an asymmetric tridiagonal matrix. The proposed procedure was based on the power method and the computation of the square of the original matrix. Then they provided numerical results with simulations in $\mathrm{C} / \mathrm{C}++$ implementation in order 
Electronic Journal of Linear Algebra, ISSN 1081-3810

A publication of the International Linear Algebra Society

Volume 36, pp. 587-598, August 2020 .

to demonstrate the effectiveness of the proposed method. They adopted the Sylvester-Kac test matrix [21] for comparing the power method and the proposed method performance. We also refer to [24] for further usage of test matrices. It is our purpose that the new matrix that we will present here and the corresponding explicit eigenvalues will make a significant contribution these type of special matrices.

2. The spectrum of $H_{n}$. In this section we prove our main result. We use basically the technique of the left eigenvectors of $H_{n}$ and an inductive approach to reach our aim.

THEOREM 2.1. The eigenvalues of $H_{n}$ are (1.1), i.e.,

$$
\{-2 \ell,-2 \ell+2, \ldots,-2,0,2, \ldots, 2 \ell-2,2 \ell\}
$$

for $n=2 \ell$, and

$$
\{-2 \ell-1,-2 \ell+1,-2 \ell+3, \ldots,-1,1, \ldots, 2 \ell-3,2 \ell-1,2 \ell+1\}
$$

for $n=2 \ell+1$.

We start by finding two eigenvalues of $H_{n}$ and then the two corresponding left eigenvectors associated to each of them.

Let us define the two $(2 n+1)$-vectors

$$
u^{+}=\left(\begin{array}{lllllll}
1 & 1 & 1 & 1 & \cdots & 1 & 1
\end{array}\right)
$$

and

$$
u^{-}=\left(\begin{array}{lllllll}
1 & -1 & 1 & -1 & \cdots & -1 & 1
\end{array}\right)
$$

The next lemma is crucial and it says that $u^{+}$and $u^{-}$are both left eigenvectors of $H_{2 n}$.

LEMmA 2.2. The matrix $H_{2 n}$ has the eigenvalues $\lambda^{+}=2 n$ and $\lambda^{-}=-2 n$ with left eigenvectors $u^{+}$and $u^{-}$, respectively.

Proof. To prove our claim, it is sufficient to show that

$$
u^{+} H_{2 n}=\lambda^{+} u^{+} \text {and } u^{-} H_{2 n}=\lambda^{-} u^{-}
$$

From the definitions of $H_{2 n}$ and $u^{+}$, we should show that

$$
\begin{aligned}
\left(u^{+} H_{2 n}\right)_{1,1} & =\left(\lambda^{+} u^{+}\right)_{1,1}, \\
\left(u^{+} H_{2 n}\right)_{1,2 n+1} & =\left(\lambda^{+} u^{+}\right)_{1,2 n+1}
\end{aligned}
$$

and

$$
\left(u^{+} H_{2 n}\right)_{1, m}=\left(\lambda^{+} u^{+}\right)_{1, m}, \quad \text { for } 1<m<2 n+1 .
$$

The first two claims are simple to check. For example, the first identity comes from

$$
\left(u^{+} H_{2 n}\right)_{1,1}=2 n=\lambda^{+}=\left(\lambda^{+} u^{+}\right)_{1,1} .
$$


Electronic Journal of Linear Algebra, ISSN 1081-3810

We now focus on the case $2 \leq k \leq 2 n$. We consider

$$
\left(u^{+} H_{2 n}\right)_{1, k}=\frac{k}{2}+\frac{4 n-k}{2}=2 n .
$$

On the other hand, the definition of $\lambda^{+}$gives

$$
\left(\lambda^{+} u^{+}\right)_{1, k}=2 n
$$

as claimed. The other case, i.e., $u^{-} H_{2 n}=\lambda^{-} u^{-}$, can be handled in a similar fashion.

Similarly to the previous case, we define two $(2 n+2)$-vectors:

$$
v^{+}=\left(\begin{array}{lllllll}
1 & 1 & 1 & 1 & \cdots & 1 & 1
\end{array}\right)
$$

and

$$
v^{-}=\left(\begin{array}{lllllll}
1 & -1 & 1 & -1 & \cdots & 1 & -1
\end{array}\right) .
$$

The next lemma can be proved analogously to the previous result.

Lemma 2.3. The matrix $H_{2 n+1}$ has the eigenvalues $\mu^{+}=2 n+1$ and $\mu^{-}=-(2 n+1)$ with left eigenvectors $v^{+}$and $v^{-}$, respectively.

For later use, we define an upper triangle matrix $U_{n}$ of order $n$ with

$$
U_{i, i}=\frac{(n-\lfloor i / 2\rfloor)(2 n+1-2\lceil i / 2\rceil)}{\left(\begin{array}{c}
n+1 \\
2
\end{array}\right)}, \quad \text { for } 1 \leq i \leq n
$$

and

$$
U_{i, i+2 r}=\frac{(n-i)(2 n+1)}{\left(\begin{array}{c}
n+1 \\
2
\end{array}\right)}, \quad \text { for } 1 \leq i \leq n-2 r \text { and } 1 \leq r \leq\left\lfloor\frac{n-1}{2}\right\rfloor
$$

and 0 , otherwise, where $\lfloor\cdot\rfloor$ and $\lceil\cdot\rceil$ stand for the floor and ceiling functions, respectively.

For example, when $n=10$, we have

$$
U_{10}=\frac{21}{55}\left[\begin{array}{cccccccccc}
\frac{190}{21} & 0 & 9 & 0 & 9 & 0 & 9 & 0 & 9 & 0 \\
0 & \frac{57}{7} & 0 & 8 & 0 & 8 & 0 & 8 & 0 & 8 \\
0 & 0 & \frac{51}{7} & 0 & 7 & 0 & 7 & 0 & 7 & 0 \\
0 & 0 & 0 & \frac{136}{21} & 0 & 6 & 0 & 6 & 0 & 6 \\
0 & 0 & 0 & 0 & \frac{40}{7} & 0 & 5 & 0 & 5 & 0 \\
0 & 0 & 0 & 0 & 0 & 5 & 0 & 4 & 0 & 4 \\
0 & 0 & 0 & 0 & 0 & 0 & \frac{13}{3} & 0 & 3 & 0 \\
0 & 0 & 0 & 0 & 0 & 0 & 0 & \frac{26}{7} & 0 & 2 \\
0 & 0 & 0 & 0 & 0 & 0 & 0 & 0 & \frac{22}{7} & 0 \\
0 & 0 & 0 & 0 & 0 & 0 & 0 & 0 & 0 & \frac{55}{21}
\end{array}\right] .
$$


Electronic Journal of Linear Algebra, ISSN 1081-3810

A publication of the International Linear Algebra Society

Volume 36, pp. 587-598, August 2020.

For an odd case as $n=11$, we have

$$
U_{11}=\frac{23}{66}\left(\begin{array}{ccccccccccc}
\frac{231}{23} & 0 & 10 & 0 & 10 & 0 & 10 & 0 & 10 & 0 & 10 \\
0 & \frac{210}{23} & 0 & 9 & 0 & 9 & 0 & 9 & 0 & 9 & 0 \\
0 & 0 & \frac{190}{23} & 0 & 8 & 0 & 8 & 0 & 8 & 0 & 8 \\
0 & 0 & 0 & \frac{171}{23} & 0 & 7 & 0 & 7 & 0 & 7 & 0 \\
0 & 0 & 0 & 0 & \frac{153}{23} & 0 & 6 & 0 & 6 & 0 & 6 \\
0 & 0 & 0 & 0 & 0 & \frac{136}{23} & 0 & 5 & 0 & 5 & 0 \\
0 & 0 & 0 & 0 & 0 & 0 & \frac{120}{23} & 0 & 4 & 0 & 4 \\
0 & 0 & 0 & 0 & 0 & 0 & 0 & \frac{105}{23} & 0 & 3 & 0 \\
0 & 0 & 0 & 0 & 0 & 0 & 0 & 0 & \frac{91}{23} & 0 & 2 \\
0 & 0 & 0 & 0 & 0 & 0 & 0 & 0 & 0 & \frac{78}{23} & 0 \\
0 & 0 & 0 & 0 & 0 & 0 & 0 & 0 & 0 & 0 & \frac{66}{23}
\end{array}\right) .
$$

A routine calculation lead us to the inverse matrix $U_{n}^{-1}=\left(C_{i j}\right)$ with the recursions

$$
\frac{C_{i, i}}{C_{i+1, i+1}}=\frac{2 n-i-1}{2 n-i+1}
$$

for $1 \leq i \leq n-1$, while, for $1 \leq r \leq\left\lceil\frac{n-2}{2}\right\rceil$,

$$
\frac{C_{i, i+2 r}}{C_{i+1, i+2 r+1}}=\frac{i+2}{i+2 r} \times \frac{n-i}{n-i-1} \times \frac{2 n-2(r+1)-i}{2 n+1-i}
$$

and 0 , otherwise, where the initials $C_{11}=\frac{n+1}{4 n-2}, C_{11} / C_{13}=(2 n-3) /(2 n+1)$ and

$$
\frac{C_{1,2 i+1}}{C_{1,2 i+3}}=\frac{(n-i-1)(2 n-2 i-3)}{h_{i+1}},
$$

for $1 \leq i \leq\left\lfloor\frac{n-3}{2}\right\rfloor$, where $h_{n}$ is the Hexagonal number defined by $h_{n}=n(2 n-1)$.

Now our purpose is to find similar matrices to $H_{2 n}$ and $H_{2 n+1}$, respectively. For this purpose, we shall give the following result.

LEMMA 2.4. The spectrum of matrix $H_{n}, \sigma\left(H_{n}\right)$, satisfy that

$$
\sigma\left(H_{2 n}\right)=\left\{\lambda^{+}, \lambda^{-}\right\} \cup \sigma\left(H_{2 n-2}\right)
$$

and

$$
\sigma\left(H_{2 n+1}\right)=\left\{\mu^{+}, \mu^{-}\right\} \cup \sigma\left(H_{2 n-1}\right)
$$

Proof. First, we consider the matrix $H_{2 n}$. Define a matrix $T$ of order $2 n+1$ as shown

$$
T=\left(\begin{array}{cc|ccccccc}
1 & 1 & 1 & 1 & 1 & 1 & \cdots & 1 & 1 \\
1 & -1 & 1 & -1 & 1 & -1 & \cdots & -1 & 1 \\
\hline & 0_{(2 n-1) \times 2} & \multicolumn{1}{c|}{I_{2 n-1}} & & &
\end{array}\right)
$$


Electronic Journal of Linear Algebra, ISSN 1081-3810

A publication of the International Linear Algebra Society

Volume 36, pp. 587-598, August 2020.

where $\mathbf{0}_{(2 n-1) \times 2}$ is the $(2 n-1) \times 2$ zero matrix and $I_{k}$ is the identity matrix of order $k$. Its inverse is

$$
T^{-1}=\left(\begin{array}{cc|cccccccc}
\frac{1}{2} & \frac{1}{2} & -1 & 0 & -1 & 0 & -1 & \cdots & 0 & -1 \\
\frac{1}{2} & -\frac{1}{2} & 0 & -1 & 0 & -1 & 0 & \cdots & -1 & 0 \\
\hline & 0_{(2 n-1) \times 2} & \multicolumn{1}{c}{I_{2 n-1}} \\
\hline
\end{array}\right.
$$

We can easily check that $H_{2 n}$ is similar to the matrix

$$
E=\left(\begin{array}{cc|c}
\lambda^{+} & 0 & \\
0 & \lambda^{-} & \mathbf{0}_{2 \times(2 n-1)} \\
\hline \frac{2 \lambda^{+}-1}{4} & \frac{2 \lambda^{-}+1}{4} & \\
& \mathbf{0}_{(2 n-2) \times 2} & W
\end{array}\right),
$$

where $W$ is the block of order $2 n-1$ defined by

$$
W=\left(\begin{array}{cccccccc}
0 & \frac{4-4 n}{2} & 0 & -\frac{4 n-1}{2} & \cdots & 0 & -\frac{4 n-1}{2} & 0 \\
\frac{4 n-2}{2} & 0 & \frac{4}{2} & & & & & \\
& \frac{4 n-3}{2} & 0 & \frac{5}{2} & & & & \\
& & \frac{4 n-4}{2} & 0 & \ddots & & & \\
& & & \frac{4 n-5}{2} & \ddots & \ddots & & \\
& & & & \ddots & \ddots & \frac{2 n-1}{2} & \\
& & & & & \frac{2 n+2}{2} & 0 & 2 n \\
& & & & & & \frac{2 n+1}{2} & 0
\end{array}\right),
$$

since $E=T H_{2 n} T^{-1}$. Consequently, $\lambda^{ \pm}$are eigenvalues of both $E$ and $H_{2 n}$.

We will study now the matrix $H_{2 n+1}$. Define the matrix $Y$ of order $2 n+2$ as

$$
Y=\left(\begin{array}{cc|ccccccc}
1 & 1 & 1 & 1 & 1 & 1 & \cdots & 1 & 1 \\
1 & -1 & 1 & -1 & 1 & -1 & \cdots & 1 & -1 \\
\hline & 0_{2 n \times 2} & \multicolumn{1}{|c}{I_{2 n}} & & &
\end{array}\right)
$$

Likewise to the previous case, we obtain

$$
Y^{-1}=\left(\begin{array}{cc|cccccccc}
\frac{1}{2} & \frac{1}{2} & -1 & 0 & -1 & 0 & -1 & \cdots & -1 & 0 \\
\frac{1}{2} & -\frac{1}{2} & 0 & -1 & 0 & -1 & 0 & \cdots & 0 & -1 \\
\hline & 0_{2 n \times 2} & \multicolumn{1}{c}{I_{2 n}} \\
& & & & & & &
\end{array}\right) .
$$


Electronic Journal of Linear Algebra, ISSN 1081-3810

A publication of the International Linear Algebra Society

Volume 36, pp. 587-598, August 2020.

Therefore, $H_{2 n+1}$ is similar to $D=Y H_{2 n+1} Y^{-1}$, where

$$
D=\left(\begin{array}{cc|c}
\mu^{+} & 0 & \\
0 & \mu^{-} & \mathbf{0}_{2 \times 2 n} \\
\hline \frac{2 \mu^{+}-1}{4} & \frac{2 \mu^{-}+1}{4} & \\
& \mathbf{0}_{(2 n-1) \times 2} & Q
\end{array}\right)
$$

and $Q$ is the matrix, of order $2 n$,

$$
Q=\left(\begin{array}{ccccccccc}
0 & \frac{2-4 n}{2} & 0 & -\frac{4 n+1}{2} & 0 & -\frac{4 n+1}{2} & \cdots & 0 & -\frac{4 n+1}{2} \\
\frac{4 n}{2} & 0 & \frac{4}{2} & & & & & & \\
& \frac{4 n-1}{2} & 0 & \frac{5}{2} & & & & & \\
& & \frac{4 n-2}{2} & 0 & \frac{6}{2} & & & & \\
& & & \frac{4 n-3}{2} & 0 & \ddots & & & \\
& & & \ddots & \ddots & \frac{2 n-1}{2} & & \\
& & & & & \frac{2 n+4}{2} & 0 & \frac{2 n}{2} & \\
& & & & & & \frac{2 n+3}{2} & 0 & 2 n+1 \\
& & & & & & & \frac{2 n+2}{2} & 0
\end{array}\right) .
$$

Thus, $\mu^{+}$and $\mu^{-}$are eigenvalues of the matrix $H_{2 n+1}$.

To compute the remaining eigenvalues of $H_{2 n+1}$ and $H_{2 n}$, we proceed providing some auxiliary results.

Taking into account the definition of $U_{n}$, we clearly have

$$
H_{2 n-2}=U_{2 n-1} W U_{2 n-1}^{-1} \quad \text { and } \quad H_{2 n-1}=U_{2 n} Q U_{2 n}^{-1}
$$

Furthermore, if we define the matrix of order $n$

$$
M_{n}=\left(\begin{array}{c|c}
I_{2} & \mathbf{0}_{2 \times(n-2)} \\
\hline \mathbf{0}_{(n-2) \times 2} & U_{n-2}
\end{array}\right),
$$

then we get

$$
M_{2 n+1} E M_{2 n+1}^{-1}=\left(\begin{array}{cc|c}
\lambda^{+} & 0 & \mathbf{0}_{2 \times(2 n-1)} \\
0 & \lambda^{-} & \\
\hline \frac{(4 n-1)(4 n-3)}{4 n} & -\frac{(4 n-1)(4 n-3)}{4 n} & U_{2 n-1}^{-1} W U_{2 n-1}
\end{array}\right)
$$


Electronic Journal of Linear Algebra, ISSN 1081-3810

and

$$
M_{2 n+2} D M_{2 n+2}^{-1}=\left(\begin{array}{cc|c}
\mu^{+} & 0 & \mathbf{0}_{2 \times(2 n-2)} \\
0 & \mu^{-} & \\
\hline \frac{(4 n-1)(4 n+1)}{2(2 n+1)} & -\frac{(4 n-1)(4 n+1)}{2(2 n+1)} & \\
\mathbf{0}_{(2 n-1) \times 2} & & U_{2 n}^{-1} Q U_{2 n}
\end{array}\right) .
$$

So far, we derived the identities

$$
\begin{aligned}
E & =T H_{2 n} T^{-1}, \\
D & =Y H_{2 n+1} Y^{-1}, \\
H_{2 n-2} & =U_{2 n-1} W U_{2 n-1}^{-1}, \\
H_{2 n-1} & =U_{2 n} Q U_{2 n}^{-1} .
\end{aligned}
$$

From the definition of $H_{n}$, both $M_{2 n+1} E M_{2 n+1}^{-1}$ and $M_{2 n+2} D M_{2 n+2}^{-1}$ can be rewritten in the following lowertriangular block form

$$
\left(\begin{array}{cc|c}
\lambda^{+} & 0 & 0 \\
0 & \lambda^{-} & \\
\hline * & H_{2 n-2}
\end{array}\right) \text { and }\left(\begin{array}{cc|c}
\mu^{+} & 0 & 0 \\
0 & \mu^{-} & \\
\hline & * & H_{2 n-1}
\end{array}\right)
$$

respectively, which give us the claimed results.

From (2.2), we derive our main result, Theorem 2.1, on the spectra of the matrix $H_{n}$.

Also for the matrix $H_{n}(x)$ defined by

$$
H_{n}(x)=\left(\begin{array}{cccccc}
x & \frac{1}{2} & & & & \\
\frac{2 n}{2} & x & \frac{2}{2} & & & \\
& \frac{2 n-1}{2} & \ddots & \ddots & & \\
& & \ddots & \ddots & \frac{n-1}{2} & \\
& & & \frac{n+2}{2} & x & n \\
& & & & \frac{n+1}{2} & x
\end{array}\right)
$$

we immediately get the recurrences on a positive integer $n$,

$$
\operatorname{det} H_{2 n+1}(x)=(x-(2 n+1))(x+(2 n+1)) \operatorname{det} H_{2 n-1}(x), \quad \text { with } \operatorname{det} H_{1}(x)=x^{2}-1,
$$

and

$$
\operatorname{det} H_{2 n}(x)=(x-2 n)(x+2 n) \operatorname{det} H_{2 n-2}(x), \quad \text { with } \operatorname{det} H_{0}(x)=x,
$$

which means that

$$
\operatorname{det} H_{2 n+1}(x)=\prod_{k=0}^{n}\left(x^{2}-(2 k+1)^{2}\right)
$$


Electronic Journal of Linear Algebra, ISSN 1081-3810

and

$$
\operatorname{det} H_{2 n}(x)=\prod_{k=0}^{n}\left(x^{2}-(2 k)^{2}\right) .
$$

3. The interlacing. Recently, it was proved in [12] the following:

THEOREM 3.1. The eigenvalues of the matrix

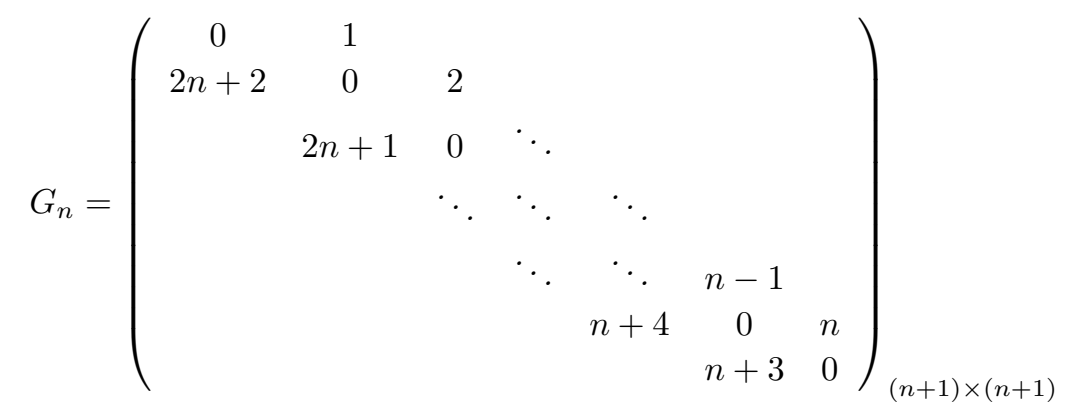

are

$$
\{ \pm 2 \bar{k}\}_{\bar{k}=0}^{n},
$$

with $\bar{k} \equiv n(\bmod 2)$. That is, they are, for $G_{2 n-1}$,

$$
\{ \pm 2, \pm 6, \pm 10, \ldots, \pm 2(2 n-1)\}
$$

while, for $G_{2 n}$, they are

$$
\{0, \pm 4, \pm 8, \pm 12, \ldots, \pm 4 n\}
$$

Notice that Theorem 3.1 says that the eigenvalues of $G_{n}$ are the double of the Sylvester-Kac matrix.

Suppose that $\hat{H}_{n}$ is the principal submatrix of order $n$ obtained from $H_{n}$ by the deletion of its last row and column. We find that $\hat{H}_{n}=\frac{1}{2} G_{n-1}$. Surprisingly, this means that $H_{n}$ is a matrix with an $n$-Sylvester spectrum with principal submatrix $\hat{H}_{n}$ with an $(n-1)$-Sylvester spectrum. Therefore, the interlacing between the eigenvalues of $H_{n}$ and $\hat{H}_{n}$ is:

$$
-n<-n+1<-n+2<\cdots<-2<-1<0<1<2<\cdots<n-2<n-1<n
$$

4. The relation between the Sylvester-Kac matrix $A_{n}$ and the new matrix $H_{n}$. Now we provide a similarity relation between the usual Sylvester-Kac matrix $A_{n}$ and the tridiagonal matrix $H_{n}$. For this, define the upper triangular matrix $T=\left(T_{i j}\right)$ of order $n$ with the recursions for terms on the band entries for $0 \leq r \leq\lfloor(n-1) / 2\rfloor$,

$$
\frac{T_{i, i+2 r}}{T_{i+1, i+2 r+1}}=\frac{i}{i+2 r} \times \frac{2 n-2 r-i-1}{2(n-r-i)},
$$

and for $0 \leq i \leq\lfloor(n-3) / 2\rfloor$,

$$
\frac{T_{1,2 i+1}}{T_{1,2 i+3}}=\frac{2 n-2 i-3}{2 i+1}
$$


and 0 , otherwise, with the initial $T_{1,1}=L(n)$, where $L(n)$ is the leading coefficient of the Legendre polynomial $P(x)$, that is,

$$
L(n)=\frac{(2 n-1) ! !}{n !},
$$

where $(2 n-1) ! !$ is the double factorial defined by $(2 n-1) ! !=1 \cdot 3 \cdot 5 \cdot \ldots \cdot(2 n-1)$.

Now, by straight computations, the inverse matrix $T^{-1}=\left(\Omega_{i j}\right)$ is given by the recursion for the elements on the bands as shown for $0 \leq r \leq\lfloor(n-1) / 2\rfloor$

$$
\frac{\Omega_{i, i+2 r}}{\Omega_{i+1, i+2 r+1}}=(-1)^{r} \frac{2 i(n-i-r-1)(n-i)}{(i+2 r)(n-i-1)(2 n-i-1)}
$$

and, for $0 \leq i \leq\lfloor(n-2) / 2\rfloor$,

$$
\frac{\Omega_{1,2 i+1}}{\Omega_{1,2 i+3}}=\frac{2(n-i-2)}{2 i+1}
$$

and 0 , otherwise, with the initial $\Omega_{1,1}=1 / L(n)$, where $L(n)$ is the leading coefficient of the Legendre polynomial $P(x)$, that is,

$$
\Omega_{1,1}=\frac{n !}{(2 n-1) ! !} .
$$

For instance, when $n=10$, we have the matrix $T$ and its inverse $T^{-1}$ as follows

$$
\begin{aligned}
& \left(\begin{array}{cccccccccc}
\frac{12155}{128} & 0 & \frac{715}{128} & 0 & \frac{143}{128} & 0 & \frac{55}{128} & 0 & \frac{35}{128} & 0 \\
0 & \frac{12155}{128} & 0 & \frac{2145}{128} & 0 & \frac{715}{128} & 0 & \frac{385}{128} & 0 & \frac{315}{128} \\
0 & 0 & \frac{715}{8} & 0 & \frac{1001}{32} & 0 & \frac{495}{32} & 0 & \frac{175}{16} & 0 \\
0 & 0 & 0 & \frac{5005}{64} & 0 & \frac{715}{16} & 0 & \frac{1925}{64} & 0 & \frac{105}{4} \\
0 & 0 & 0 & 0 & \frac{1001}{16} & 0 & \frac{825}{16} & 0 & \frac{175}{4} & 0 \\
0 & 0 & 0 & 0 & 0 & \frac{715}{16} & 0 & \frac{385}{8} & 0 & \frac{189}{4} \\
0 & 0 & 0 & 0 & 0 & 0 & \frac{55}{2} & 0 & 35 & 0 \\
0 & 0 & 0 & 0 & 0 & 0 & 0 & \frac{55}{4} & 0 & 18 \\
0 & 0 & 0 & 0 & 0 & 0 & 0 & 0 & 5 & 0 \\
0 & 0 & 0 & 0 & 0 & 0 & 0 & 0 & 0 & 1
\end{array}\right)^{-1} \\
& =\left(\begin{array}{cccccccccc}
\frac{128}{12155} & 0 & -\frac{8}{12155} & 0 & \frac{12}{85085} & 0 & -\frac{1}{17017} & 0 & \frac{1}{24310} & 0 \\
0 & \frac{128}{12155} & 0 & -\frac{192}{85085} & 0 & \frac{16}{17017} & 0 & -\frac{8}{12155} & 0 & \frac{9}{12155} \\
0 & 0 & \frac{8}{715} & 0 & -\frac{4}{715} & 0 & \frac{3}{715} & 0 & -\frac{7}{1430} & 0 \\
0 & 0 & 0 & \frac{64}{5005} & 0 & -\frac{64}{5005} & 0 & \frac{12}{715} & 0 & -\frac{24}{715} \\
0 & 0 & 0 & 0 & \frac{16}{1001} & 0 & -\frac{30}{1001} & 0 & \frac{10}{143} & 0 \\
0 & 0 & 0 & 0 & 0 & \frac{16}{715} & 0 & -\frac{56}{715} & 0 & \frac{252}{715} \\
0 & 0 & 0 & 0 & 0 & 0 & \frac{2}{55} & 0 & -\frac{14}{55} & 0 \\
0 & 0 & 0 & 0 & 0 & 0 & 0 & \frac{4}{55} & 0 & -\frac{72}{55} \\
0 & 0 & 0 & 0 & 0 & 0 & 0 & 0 & \frac{1}{5} & 0 \\
0 & 0 & 0 & 0 & 0 & 0 & 0 & 0 & 0 & 1
\end{array}\right) .
\end{aligned}
$$

Now we can give our latest main result without proof which follows from matrix multiplication. 
THEOREM 4.1. The similarity relation between the Sylvester-Kac matrix $A_{n-1}$ and the matrix $H_{n-1}$ can be given by

$$
A_{n-1}=T H_{n-1} T^{-1}
$$

For example, when $n=9$, we have that

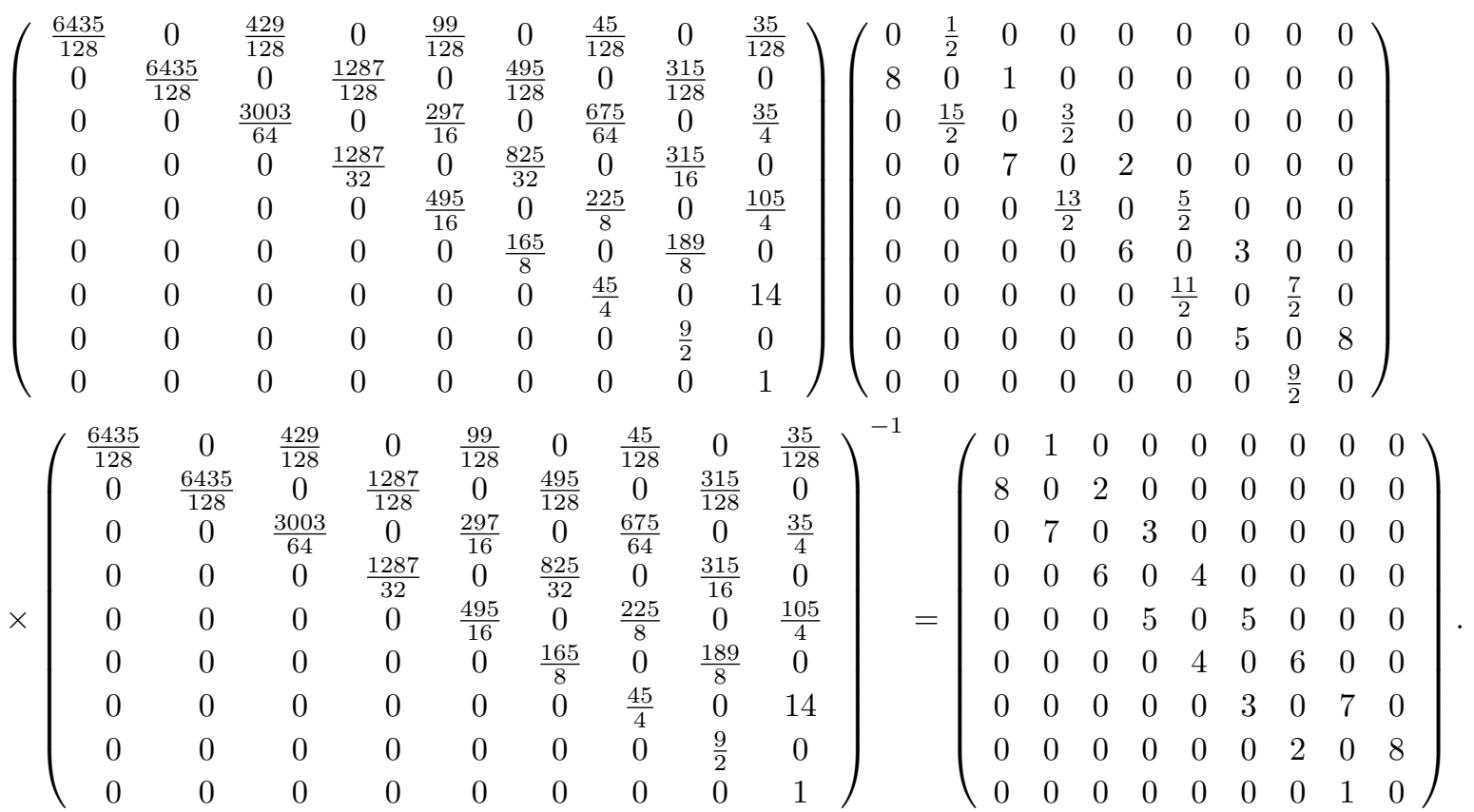

\section{REFERENCES}

[1] R. Askey. Evaluation of Sylvester type determinants using orthogonal polynomials. In: H.G.W. Begehr et al. (editors), Advances in Analysis. Hackensack, NJ, World Scientific, 1-16, 2005.

[2] R. Askey and J. Wilson. A set of orthogonal polynomials that generalize the Racah coefficients or $6-j$ symbols. SIAM Journal on Mathematical Analysis, 10:1008-1016, 1979.

[3] T. Boros and P. Rózsa. An explicit formula for singular values of the Sylvester-Kac matrix. Linear Algebra and its Applications, 421:407-616, 207.

[4] A.E. Brouwer, A.M. Cohen, and A.Neumaier. Distance-Regular Graphs. Ergebnisse der Mathematik und ihrer Grenzgebiete, 18, Springer-Verlag, New York, 1989.

[5] T.S. Chow. A class of Hessenberg matrices with known eigenvalues and inverses. SIAM Review, 11:391-395, 1969.

[6] W. Chu. Fibonacci polynomials and Sylvester determinant of tridiagonal matrix. Applied Mathematics and Computation, 216:1018-1023, 2010.

[7] W. Chu and X. Wang. Eigenvectors of tridiagonal matrices of Sylvester type. Calcolo, 45:217-233, 2008.

[8] D.F.G. Coelho, V.S. Dimitrov, and L. Rakai. Efficient computation of tridiagonal matrices largest eigenvalue. Journal of Computational and Applied Mathematics, 330:268-275, 2018.

[9] P.A. Clement. A class of triple-diagonal matrices for test purposes. SIAM Review, 1:50-52, 1959.

[10] A. Edelman and E. Kostlan. A class of triple-diagonal matrices for test purposes. In: J. Lewis (editor), Proceedings of the Fifth SIAM Conference on Applied Linear Algebra, SIAM, Philadelpia, 503-507, 1994.

[11] C.M. da Fonseca. A short note on the determinant of a Sylvester-Kac type matrix. International Journal of Nonlinear Sciences and Numerical Simulation, DOI:10.1515/ijnsns-2018-0375. 
[12] C.M. da Fonseca and E. Kılıç. A new type of Sylvester-Kac matrix and its spectrum. Linear and Multilinear Algebra, DOI:10.1080/03081087.2019.1620673.

[13] C.M. da Fonseca and E. Kılıç. An observation on the determinant of a Sylvester-Kac type matrix. Analele Universitatii "Ovidius" Constanţa - Seria Matematica, 28:111-115, 2020.

[14] C.M. da Fonseca, D.A. Mazilu, I. Mazilu, and H.T. Williams. The eigenpairs of a Sylvester-Kac type matrix associated with a simple model for one-dimensional deposition and evaporation. Applied Mathematics Letters, 26:1206-1211, 2013.

[15] O. Holtz. Evaluation of Sylvester type determinants using block-triangularization. In: H.G.W. Begehr et al. (editors), Advances in Analysis, Hackensack, NJ, World Scientific, 395-405, 2005.

[16] Kh.D. Ikramov. On a remarkable property of a matrix of Mark Kac. American Mathematical Monthly, 72:325-330, 2002.

[17] M. Kac. Random walk and the theory of Brownian motion. American Mathematical Monthly, 54:369-391, 1947.

[18] E. Kılıç. Sylvester-tridiagonal matrix with alternating main diagonal entries and its spectra. International Journal of Nonlinear Sciences and Numerical Simulation, 14:261-266, 2013.

[19] E. Kılıç and T. Arıkan. Evaluation of spectrum of 2-periodic tridiagonal-Sylvester matrix. Turkish Journal of Mathematics, 40:80-89, 2016.

[20] T. Muir. The Theory of Determinants in the Historical Order of Development, Vol. II. Dover Publications Inc., New York, 1960.

[21] R. Oste and J. Van den Jeugt. Tridiagonal test matrices for eigenvalue computations: Two-parameter extensions of the Clement matrix. Journal of Computational and Applied Mathematics, 314:30-39, 2017.

[22] P. Rózsa. Remarks on the spectral decomposition of a stochastic matrix. Magyar Tudomanyos Akademia. Matematikai es Fizikai Tudomanyok Osztalyanak Közlemenyei, 7:199-206, 1957.

[23] E. Schrödinger. Quantisierung als Eigenwertproblem III. Annalen der Physik, 80:437-490, 1926.

[24] T. Suzuki and T. Suzuki. An eigenvalue problem for derogatory matrices. Journal of Computational and Applied Mathematics, 199:245-250, 2007.

[25] J.J. Sylvester. Théorème sur les déterminants. Nouvelles Annales de Mathématiques, 13:305, 1854.

[26] O. Taussky and J. Todd. Another look at a matrix of Mark Kac. Linear Algebra and its Applications, 150:341-360, 1991.

[27] I. Vincze. Über das Ehrenfestsche Modell der Wärmeübertragung. Archiv der Mathematik, 15:394-400, 1964. 